Aim of the study: Prostate cancer is the second most prevalent cancer among men in Poland. The main methods of radical treatment are radical prostatectomy and radiotherapy. In patients who have been correctly qualified for surgery, a positive surgical margin is always an unexpected and undesirable factor. The aim of this prospective study was to evaluate the incidence of positive margins in more than 100 consecutive patients with prostate cancer undergoing radical prostatectomy.

Material and methods: The study included 114 patients aged 44-78 years (mean 61.5 years) who underwent surgery for prostate cancer in stage cT13NO/MO (according to the TNM staging system) in the years 2010-2011 in the Clinical Department of Oncological Urology in the Center of Oncology in Bydgoszcz.

Results: The presence of positive surgical margins was found in $45(39.47 \%)$ patients, and in $20(17.54 \%)$ margins were assessed as close (1-2 mm). Among the patients with positive surgical margins about $22 \%$ had biochemical recurrence. Among patients with negative surgical margins $13 \%$ of pT $2 \mathrm{c}$ and $12.5 \%$ of pT3a had biochemical recurrence. Patients with positive surgical margins, along with patients diagnosed with tumor extending beyond the prostate (pT3a) or invading seminal vesicles (pT3b), are at an increased risk of recurrence and progression, reaching up to $30-50 \%$ over 10 years. The risk is $2-4$ times higher than in patients without positive operating margins.

Key words: prostate cancer, operation, positive margins.

Contemp Oncol (Pozn) 2013; 17 (4): 383-388 DOI: $10.5114 /$ wo.2013.37217

\section{Indications for postoperative radiotherapy in patients with prostate cancer after surgery with positive surgical margins}

\author{
Krzysztof Kamecki', Marta Biedka ${ }^{2,3}$, Roman Makarewicz ${ }^{2}$, Jerzy Siekiera ${ }^{1}$
}

${ }^{1}$ Clinical Department of Oncological Urology, The Franciszek Lukaszczyk Oncology Center, Bydgoszcz, Poland

${ }^{2}$ Chair and Clinic of Oncology and Brachytherapy, Ludwik Rydygier Collegium Medicum Bydgoszcz, Nicolaus Copernicus University, Torun, Poland

${ }^{3}$ Radiotherapy Department 1, Oncology Centre, Bydgoszcz, Poland

\section{Introduction}

Prostate cancer is the second most prevalent cancer among men in Poland. The etiopathogenesis of prostate cancer, like most other cancer types, is obscure. Among the risk factors there are age, race and genetics [1]. Over $95 \%$ of prostate cancers are adenocarcinomas, which arise most frequently in the peripheral zone of the prostate. The first metastases occur in pelvic lymph nodes and bone. The most important prognostic factors for prostate cancer include TNM stage, histopathological classification (Gleason score), PSA level, the status of the lymph nodes and evaluation of distant metastases [2].

Selection of an optimal treatment method for patients with prostate cancer depends on the stage of the disease as well as the patient's life expectancy and general quality of life. In the case of organ-confined cancer radical treatment is possible. The main methods of radical treatment are radical prostatectomy and radiotherapy [1-3]. Both methods are used alone or in combination $[4,5]$. The predictors of treatment efficacy, useful in observing patients undergoing radical prostatectomy, include post-operative PSA level, TNM stage, Gleason score and surgical margin status. Apart from the last factor, the others are related to tumor characteristics and are independent of the operator. Since in patients who have been correctly qualified for surgery a positive surgical margin is always an unexpected and undesirable factor, surgical margin status can be considered as a criterion for the quality of surgery. The percentage of positive margins is related to the surgeon's experience (unintended injury of prostate capsule during treatment of the tumor not extending beyond the capsule), tumor characteristics (tumor infiltration beyond the capsule), anatomical features of the patient (patient's obesity causing technical difficulties during the preparation of the prostate located deep in the pelvis minor), and the way of processing the material during histopathological evaluation (false positive margins) [2].

The presence of positive surgical margins can imply incomplete excision of malignant tissue and is an indication for adjuvant therapy. However, in contrast to non-radical surgical treatment of solid tumors in other organs, in the case of prostate cancer with positive surgical margins, there is no standard regime. According to some investigators, the presence of positive surgical margins does not necessarily mean a worse prognosis and is not in each case an indication for adjuvant treatment.

\section{Aim of the study}

The aim of this prospective study was to evaluate the incidence of positive margins in more than 100 consecutive patients with prostate cancer undergo- 
Table 1. Study group characteristics

\begin{tabular}{|c|c|c|c|}
\hline Clinical stage & cT1 & $\mathrm{cT} 2$ & cT3 \\
\hline $\begin{array}{l}\text { Number of patients who underwent radical } \\
\text { prostatectomy }\end{array}$ & $\begin{array}{c}51 \\
44.7 \% \text { of the group }\end{array}$ & $\begin{array}{l}44 \\
38.6 \% \text { of the group }\end{array}$ & $\begin{array}{c}19 \\
16.7 \% \text { of the group }\end{array}$ \\
\hline Gleason score on prostate biopsy & $\begin{array}{c}2-8 \\
\text { mean } 5.98\end{array}$ & $\begin{array}{c}5-9 \\
\text { mean } 6.06\end{array}$ & $\begin{array}{c}5-8 \\
\text { mean } 6.37\end{array}$ \\
\hline Preoperative PSA level in the blood $[\mathrm{ng} / \mathrm{ml}]$ & $\begin{array}{l}3.09-53.00 \\
\text { mean } 9.87\end{array}$ & $\begin{array}{l}3.44-28.30 \\
\text { mean } 9.32\end{array}$ & $\begin{array}{l}1.06-60.00 \\
\text { mean } 20.99\end{array}$ \\
\hline Patient age [years] & $\begin{array}{c}44-71 \\
\text { mean } 61.27\end{array}$ & $\begin{array}{c}51-78 \\
\text { mean } 62.75\end{array}$ & $\begin{array}{c}51-70 \\
\text { mean } 59.58\end{array}$ \\
\hline
\end{tabular}

Table 2. Study group Gleason score

$\begin{array}{llllllllll}\text { Gleason score } & 2 & 3 & 4 & 5 & 6 & 7 & 8 & 9 \\ \text { Number of patients } & 0 & 0 & 1 & 18 & 66 & 17 & 9 & 3\end{array}$

ing radical prostatectomy. We analyzed the early results of surgical treatment and procedures followed by doctors qualifying patients with positive surgical margins for adjuvant treatment. Based on published research we present rules for qualifying patients for this treatment, as followed by other authors.

\section{Material and methods}

The study included 114 patients aged $44-78$ years (mean 61.5 years) who underwent surgery for prostate cancer in stage cT1-3NO/MO (according to the TNM staging system) in the years 2010-2011 in the Clinical Department of Oncological Urology in the Center of Oncology in Bydgoszcz. Descriptive data of the tested group of patients are presented in Tables 1 and 2. Each patient was treated in accordance with the standard procedures for prostate cancer and each patient was informed about the research and signed informed consent on participation.

Radical retropubic prostatectomy involved removing the prostate with the tumor, the seminal vesicles and iliac and obturator lymph nodes. Following the outcome of the histopathological examination, patients were directed to adjuvant therapy (radiotherapy and/or hormone therapy) in the case of the presence of at least one of the risk factors for recurrence: stage PT3 carcinoma of the prostate, the presence of lymph node metastases ( $\mathrm{pN1}$ ), or incomplete excision of malignant tissue.

Teleradiotherapy involved radiation of the prostate bed including a margin of healthy tissue (total dose of 60-74 Gy in 2 Gy daily fractions). For patients with confirmed lymph node metastases, in the first phase radiation was directed to the pelvic area, including the area of obturator lymph nodes, presacral lymph nodes, external, internal and common iliac lymph nodes to 45-46 Gy dose, and the next step was increasing the dose to the prostate bed to 60-74 Gy. The first follow-up visit took place 4-6 weeks after surgery, and subsequent visits took place at 3-month intervals. Follow-up ranges from 4 to 18 months after surgery.

\section{Results}

The study was conducted in a group of 114 patients aged 44-78 years (mean 61.5 years) who underwent radical sur- gical treatment of adenocarcinoma of the prostate. Gleason score was evaluated based on biopsy before treatment initiation. On baseline testing mean PSA level in the blood was $11.74 \mathrm{ng} / \mathrm{ml}(1.06-60 \mathrm{ng} / \mathrm{ml})$. On follow-up PSA testing 3 months after surgical treatment, median PSA level was 0.226 $\mathrm{ng} / \mathrm{ml}(0.002-8.7 \mathrm{ng} / \mathrm{ml})$. Mean follow-up was 12 months (range 6 to 18). During this period, a biochemical relapse after radical treatment (PSA $>0.2 \mathrm{ng} / \mathrm{ml}$ ) occurred in $16.36 \%$ of the patients (patients with pN1 were excluded, as the resection was recognized as incomplete). All recurrences occurred within 6 months after surgery. PSA concentration in the blood of patients with biochemical relapse after surgery was above $0.2 \mathrm{ng} / \mathrm{ml}$, with a growing trend. Biochemical progression also occurred in all patients with lymph node metastases.

The presence of positive surgical margins was found in 45 (39.47\%) patients, and in 20 (17.54\%) margins were assessed as close (1-2 mm). Among the patients with positive surgical margins about $22 \%$ had biochemical recurrence.

Among patients with negative surgical margins $13 \%$ of pT2c and $12.5 \%$ of pT3a had biochemical recurrence. Given the histopathological evaluation and Gleason score, recurrence concerned $15.47 \%$ of patients with Gleason score $<7$, $14.28 \%$ of Gleason score $=7$, and $27.27 \%$ of Gleason score $>7$. Five (4.39\%) patients had lymph node metastases. Since the surgical treatment in these patients could not be recognized as radical, this group of patients was excluded from the statistics relating to the incidence of positive margins and biochemical recurrence. Data on the early postoperative results of patients are shown in Table 3.

\section{Discussion}

The aim of radical surgery is removal of the prostate along with the malignant tumor, seminal vesicles and lymph nodes in such a way that surgical margins are negative and urinary continence and potency maximally preserved. The only objective criterion of how radical the prostatectomy was is the lack of positive surgical margins in the histopathological evaluation.

The presence of positive surgical margins always raises the question of whether the surgeon performed the operation correctly and whether the presence of positive mar- 
Table 3. Postoperative study group characteristics

\begin{tabular}{|c|c|c|c|c|c|c|c|}
\hline Clinical stage prior to surgery & cT1 & \multicolumn{2}{|c|}{ cT2 } & \multicolumn{3}{|c|}{ cT3 } & Total \\
\hline Number of patients who underwent prostatectomy & 52 & \multicolumn{2}{|c|}{43} & \multicolumn{3}{|c|}{19} & 114 \\
\hline Postoperative pathological stage $\mathrm{pT} 2 \mathrm{a}$ & pT2a & pT2b & pT2c & pT3a & pT3b & pT4a & \\
\hline Number of patients who underwent prostatectomy & $\begin{array}{c}7 \\
6.14 \%\end{array}$ & $\begin{array}{c}4 \\
3.51 \%\end{array}$ & $\begin{array}{c}64 \\
56.14 \%\end{array}$ & $\begin{array}{c}14 \\
12.28 \%\end{array}$ & $\begin{array}{c}24 \\
21.05 \%\end{array}$ & $\begin{array}{c}1 \\
0.88 \%\end{array}$ & $\begin{array}{c}114 \\
100 \%\end{array}$ \\
\hline Number of pN1 patients & 0 & 0 & 0 & 0 & 4 & 1 & 5 \\
\hline Number of pN1 patients with positive surgical margins & 0 & 0 & 0 & 0 & 4 & 1 & 5 \\
\hline Number of patients with negative surgical excluding pN1 patients & 7 & 4 & 46 & 9 & 3 & 0 & 69 \\
\hline Number of patients with positive surgical excluding pN1 patients & 0 & 0 & 18 & 5 & 17 & 0 & 40 \\
\hline Number of patients with close margins & 0 & 1 & 15 & 2 & 2 & 0 & 20 \\
\hline Number of patients with biochemical recurrence, excluding pN1 patients & s 1 & 0 & 9 & 2 & 6 & 0 & 18 \\
\hline Including number of patients with positive margins & 0 & 0 & 3 & 1 & 6 & 0 & 10 \\
\hline Including number of patients with close margins & 0 & 0 & 2 & 0 & 0 & 0 & 2 \\
\hline Including number of patients with negative margins & 1 & 0 & 6 & 1 & 0 & 0 & 8 \\
\hline Postoperative RT alone - number of patients & 0 & 0 & 7 & 9 & 16 & 0 & 32 \\
\hline Including patients pN1 & 0 & 0 & 0 & 0 & 1 & 0 & 1 \\
\hline Including patients with positive margins & 0 & 0 & 4 & 4 & 13 & 0 & 21 \\
\hline Including patients with close margins & 0 & 0 & 0 & 1 & 1 & 0 & 2 \\
\hline Postoperative RT combined with HT - number of patients & 0 & 0 & 3 & 0 & 9 & 0 & 12 \\
\hline Including pN1 patients & 0 & 0 & 0 & 0 & 3 & 0 & 3 \\
\hline Postoperative HT alone & 1 & 0 & 1 & 0 & 0 & 1 & 3 \\
\hline Including $\mathrm{pN} 1$ patients & 0 & 0 & 0 & 0 & 0 & 1 & 1 \\
\hline
\end{tabular}

gins will affect the outcome of the disease and its treatment [6]. Unfortunately, despite the improvement of surgical techniques positive margins after surgery occur in an average of $28 \%$ of patients with organ-confined ( $\mathrm{pT} 2$ ) prostate cancer as a result of preparation of the prostate in the wrong anatomical plane [7, 8], and in up to $46.5 \%$ of patients with non-organ confined (pT3) prostate cancer [9]. Patients with positive surgical margins, along with patients diagnosed with tumor extending beyond the prostate (pT3a) or invading seminal vesicles (pT3b), are at an increased risk of recurrence and progression, reaching up to $30-50 \%$ over 10 years. The risk is 2-4 times higher than in patients without positive operating margins [10].

According to large multicenter statistical analyses, the percentage of patients with increased risk factors for prostate cancer recurrence after surgery is up to 50\% [11]. These data are of potential concern to urologists performing radical prostatectomy, but it should also be pointed out that not all patients with positive surgical margins will have cancer recurrence. At least two conclusions can be drawn from this observation: the presence of a positive surgical margin does not always mean leaving the cancerous tissue behind in the site of surgery, and a positive surgical margin is not the only factor affecting the prognosis.

Ohori et al. confirm this, demonstrating that surgical margin status is an independent prognostic factor only for pT3a patients, unlike pT2 and pT3b patients. They point out that prostate-confined tumors, especially the early-stage disease (pT2a-pT2b), have a very good prognosis regardless of the state of the surgical margin, whereas advanced tumors, infiltrating the seminal vesicles (pT3b) or with lymph node metastases, have poor prognosis irrespective of surgical margin status [12].

The incidence of positive surgical margins in patients undergoing surgical treatment is directly related to preoperative PSA level > $10 \mathrm{ng} / \mathrm{ml}$, tumor extending beyond the prostate ( $\mathrm{pT} 3$ ), Gleason score, the volume of tumor in the prostate, the presence of vascular invasion into the nerve bundles and emboli made of cancer cells in the lymph vessels, applying techniques saving neurovascular bundles, smaller prostate volume, and increasing BMI [10, 13-15]. Pelvic MRI performed prior to surgery in patients with suspected infiltration outside the prostatic capsule confirmed neither protective significance of positive margins nor increase in their incidence. Causes of positive surgical margins are presented in Table 4.

Much of today's research confirms not only a greater likelihood of recurrence in patients with pT2 and iatrogenic damage to the prostate capsule than in patients without this damage, but also similar to or greater than $\mathrm{PT} 3$ patients but with negative surgical margins [16]. It is worth noting that even $28 \%$ of post-operative histopathological examination shows 
Table 4. Causes of positive surgical margins after radical prostatectomy

\section{Causes of positive surgical margins after radical prostatectomy}

1. Iatrogenic, surgical damage to the prostate, which reaches the tumor in patients with organ-confined cancer (pT2)

2. Surgical incision through infiltration that extends beyond the confines of the prostate gland (pT3)

3. Incorrect qualification of patients for surgical techniques aimed at preserving neurovascular bundles

4. The use of techniques of bladder neck preservation with a view to improving urinary incontinence [4]

5. Artifact resulting from improper handling of specimen during prostatectomy or during preparation of specimen for histopathological evaluation

the presence of prostate cancer in the lobe in which the preoperative biopsy failed to detect the tumor. The surgical technique for neurovascular bundle preservation on the side of the lobe which in the biopsy evaluation did not contain tumor results in the presence of a positive surgical margin in 4\% of surgeries, compared to $8 \%$ of cases in which cancer was found in the lateral lobe before prostatectomy [10].

Biochemical recurrence after radical prostatectomy in $\mathrm{pT} 2$ patients with positive margins is observed almost twice as frequently as in patients without positive surgical margins; it also occurs earlier and proceeds more rapidly in patients with a higher Gleason score $[4,16]$. In long-term observation it was found that the risk of biochemical recurrence was similar in pT2 patients with positive surgical margins and pT3 patients without positive surgical margins approximately 9 years after prostatectomy $[6,13]$. Therefore, a modification of the TNM staging system has been postulated, suggesting the inclusion of patients with organ-confined prostate cancer and positive surgical margins to group pT3 [17]. The risk of recurrence is not related to the location or size of positive surgical margins [13]. In pT3 patients, in turn, infiltration beyond the prostate is such a strong risk factor for recurrence that the presence of positive surgical margins in the postoperative specimen no longer has clinical significance [13].

Some authors have demonstrated that postoperative radiotherapy mainly benefits patients with $\mathrm{pT} 2$ and positive surgical margins, not pT3 patients [13]. However, the use of adjuvant radiation therapy in patients with positive margins and biochemical progression is widely recognized as standard of care, regardless of the stage of the tumor.

The recognition of positive surgical margins as an independent prognostic factor for recurrence of cancer is still controversial and results in a lack of established standard procedure in patients with localized prostate cancer and positive margins after surgery [13].

Table 5. Predictors of high-risk prostate cancer recurrence after surgery with positive surgical margins and extracapsular invasion pT3a

Predictors of high-risk prostate cancer recurrence after surgery with positive surgical margins and extracapsular invasion pT3a

Patient age $>60$ years

Preoperative PSA level $>10 \mathrm{ng} / \mathrm{ml}$

Gleason score 4 + 3, 8-10

Two or more positive surgical margins

Surgical specimen weight after prostatectomy $<30 \mathrm{~g}$
Published reports informing that only about 30\% of patients in this group have a biochemical recurrence and $20 \%$ experience local recurrence can be hypothetically explained with the effect of electrocautery during surgery, vascular damage in the site of positive margin or the effect of the inflammatory response associated with healing [8].

Higher risk of recurrence in patients with pT2 and positive surgical margins correlates with increasing Gleason grade. According to some authors, this allows one to extract a group with a low risk of recurrence, which includes patients with a preoperative PSA level $<10 \mathrm{ng} / \mathrm{ml}$ and Gleason score 2-6. Patients from this risk group may be put under observation, where PSA $>0.4 \mathrm{ng} / \mathrm{ml}$ would be considered as the criterion for recurrence and exceeding that level would be an indication for radiotherapy. Such a procedure aims at limiting the indications for postoperative radiotherapy, which carries the risk of complications and significantly increases the cost of treatment. The proposal to recognize the recurrence criterion PSA $>0.4 \mathrm{ng} / \mathrm{ml}$ is due to a number of observational studies in which it was found that PSA levels below this value in patients with positive margins who will not have recurrence tend to be stable and not likely to progress. Observation and using adjuvant radiotherapy after exceeding the threshold PSA $=0.4 \mathrm{ng} / \mathrm{ml}$ best identifies patients who actually have a recurrence of cancer and allows one to avoid the risk of complications and cost of radiation therapy for patients who have positive surgical margins but never experience recurrence $[18,19]$. Even more remarkably, the latter group includes patients who underwent surgery aimed at preserving potency and good urinary continence, which would be largely wasted on the use of radiotherapy. The trend towards reducing the toxicity of adjuvant treatment and its use only in patients with the highest risk of recurrence may help to modify the current procedure so that not only the patients with positive surgical margins and pT2 stage, but also selected patients with pT3a prostate cancer would be referred for observation. According to current guidelines, all patients in stage pT3a are referred for radiotherapy. The criteria for deferral of adjuvant therapy in patients with positive margins and/or infiltration beyond the prostate gland but without invasion of seminal vesicles and lymph nodes are shown in Table 5 [20].

It is quite a popular view that a positive surgical margin means leaving a small number of non-vascularised cancer cells and the resulting possible recurrence can be quickly and reliably identified on the basis of increase in PSA. However, the decision to postpone the adjuvant treatment of patients 
with positive surgical margins and extracapsular invasion pT3a is difficult, especially as some research points to better prognosis of patients with prostate cancer in stage pT3a and positive margins involving prolonging the biochemical recurrencefree period by $60 \%$, resulting from immediate radiation therapy after surgery $[8,21]$.

In recent years there have been significant advances in the technology of radiation, thus allowing for more efficient use of the radiation dose, while reducing side effects. In the SWOG 8794 study conducted among 431 patients with pNOMO after surgery, diagnosed with extraprostatic infiltration, positive margins or seminal vesicle invasion, observation or adjuvant radiotherapy was applied at a dose of 6064 Gy. It has been shown to improve the overall 15-year survival by $10 \%$ from $37 \%$ to $47 \%$, and distant metastasisfree survival also improved from $38 \%$ to $46 \%$. The study also assessed biochemical recurrence, finding that it occurred in a smaller group of patients (55\% vs. $77 \%$ ), whereas local failure decreased from $22 \%$ to $8 \%$. In addition, research on a group of 217 patients evaluated the quality of life and showed a higher percentage of dysfunction of the bladder in patients who received radiation therapy, but this difference disappeared after a period of 2 years after termination of treatment. The authors did not demonstrate differences in sexual dysfunction between the group undergoing observation and the group with adjuvant radiotherapy [22].

The authors of the EORTC 22911 study evaluated a group of 1005 patients with pNO with infiltration beyond the capsule of the prostate, positive margins or seminal vesicle invasion. Half the group underwent observation and the remainder were treated with radiotherapy at a dose of $60 \mathrm{~Gy}$. The groups showed no difference in the overall 5-year survival, but the authors observed a higher rate of biochemical progression-free survival (74\% vs. 53\%) and of clinical progressionfree survival ( $85 \%$ vs. $77 \%$ ). Local failure was lower in the irradiated group, accounting for $5 \%$ vs. $15 \%$. The researchers have demonstrated the predictive and prognostic value of the benefit of radiotherapy in patients with positive margins [23].

Although the incidence of positive surgical margins in histopathological assessment can be treated as a criterion for the evaluation of urological performance, there are no universally accepted standards for detection of positive margins. The method of preparation of specimens for histopathological evaluation may have as great an impact on the incidence of positive margins as the experience of the operator. In the case of an experienced surgeon, the incidence of positive margins, assessed by different pathological laboratories, can vary by $20 \%$ [19]. Differences are due to different standards of histopathological specimen preparation, experience of the histopathologist, workload and time spent on assessment. So far there has been no success in creating a classification of positive surgical margins and surgical experience suggests that the distinction between broad and focal positive margins will have different meaning for the prognosis of the patient. With this in mind it should also be taken into account that the decision about observation vs. adjuvant treatment, and the detection or non-detection of positive surgical margins, may not be based on fully reliable data.

\section{Summary}

The prognosis in patients after surgical treatment of prostate cancer based only on surgical margins will distinctly demonstrate that the presence of positive margins always implies worse prognosis. This is due to the fact that positive surgical margins are present primarily in patients with locally advanced disease and a high Gleason score, whereas no positive margins occur mostly in patients with organ-confined disease in which the prognosis is significantly better. Many studies have demonstrated that surgical margin status affects biochemical recurrence, but only large scale studies of patients after radical prostatectomy, assessing the results of treatment in a sufficiently long follow-up period, show the impact of surgical margins on prostate cancer mortality. In a study of a group of more than 65,000 patients it was demonstrated that the presence of positive surgical margins increases the risk of death from prostate cancer by 2.6 times [24]. In multivariate analyses, it has been proven that surgical margin status is an independent predictor of death from prostate cancer. This points to the necessity of improving the surgical technique to achieve the best oncological results.

In recent years, centers with vast experience in performing radical prostatectomy have reported a decreasing percentage of operations with positive surgical margins and have set the standard below $10 \%[21,25]$. This is probably a consequence of three factors: changes in surgical technique, surgeon experience and increased detection of early-stage prostate cancer. Since in $\mathrm{pT} 2$ patients with positive margins and undetectable PSA after surgery postoperative radiotherapy has been shown to double biochemical recurrence-free survival with minimal local toxicity, referring patients with positive surgical margins for adjuvant radiotherapy should be considered a standard [21].

The authors declare no conflict of interest.

\section{References}

1. Kulik A, Dąbkowski M. Radioterapia raka gruczołu krokowego. Wspolczesna Onkol 2011; 15: 317-22.

2. Cieśliński P, Hojan-Osicka M, Antczak A, Kwias Z. Assessment of intraoperative and postoperative complications during implementation of laparoscopic radical prostatectomy in patients with locally advanced prostate cancer. Wspolczesna Onkol 2011; 15: 372-5.

3. Makarewicz R, Lebioda A, Terlikiewicz J, Biedka M, Wiśniewski T. PSA bouncing after brachytherapy HDR and external beam radiotherapy a study of 121 patients with minimal 5-years follow-up. J Contemp Brachyther 2009; 1: 92-6.

4. Milecki P, Kwias Z, Martenka DJ. Radiotherapy combined with hormonal therapy $(\mathrm{RH}-\mathrm{HT})$ in prostate cancer patients with low, intermediate, and high risk of biochemical recurrence and therapeutic gain analysis. Neoplasma 2007; 54: 7-15.

5. Soumarová R, Homola L, Stursa M, Perková H. Acute toxicity of conformal high dose interstitial brachytherapy boost in prostate cancer. Neoplasma 2006; 53: 410-7.

6. Paul R, Hoppmann M, van Randenborgh H, Kübler H, Alschibaja M, Günther M, Hartung R. Residual benign prostatic glands at the urethrovesical anastomosis after radical retropubic prostatectomy. Eur Urol 2004; 46: 321-6. 
7. Bianco FJ, Grignon DJ, Sakr WA, Shekarriz B, Upadhyay J, Dornelles E, Pontes JE. Radical prostatectomy with bladder neck preservation: impact on positive margins. Eur Urol 2003; 43: 461-6.

8. Vis AN, Schröder FH, van der Kwast TH. The actual value of the surgical margin status as a predictor of disease progression in men with early prostate cancer. Eur Urol 2006; 50: 258-65.

9. Swindle P, Eastham JA, Ohori M, Kattan MW, Wheeler T, Maru N, Slawin K, Scardino PT. Do margins matter? The prognostic significance of positive surgical margins in radical prostatectomy spec imens. J Urol 2005; 174: 903-7.

10. Secin FP, Serio A, Bianco FJ Jr, Karanikolas NT, Kuroiwa K, Vickers A Touijer K, Guillonneau B. Preoperative and intraoperative risk fac tors for side-specific positive surgical margins in laparoscopic radical prostatectomy for prostate cancer. Eur Urol 2007; 51: 764-71.

11. Hoffman KE, Nguyen PL, Chen MH, Chen RC, Choueiri TK, Hu JC, Kuban DA, D'Amico AV. Recommendations for post-prostatectomy radiation therapy in the United States before and after the pre sentation of randomized trials. J Urol 2011; 185: 116-20.

12. Graefen M. The positive surgical margin after radical prostatectomy - why do we still not really know what it means? Eur Urol 2006; 50: 199-201.

13. Psutka SP, Feldman AS, Rodin D, Olumi AF, Wu CL, McDougal WS. Men with organ-confined prostate cancer and positive surgical margins develop biochemical failure at a similar rate to men with extracapsular extension. Urology 2011; 78: 121-5.

14. Palisaar RJ, Noldus J, Graefen M, Erbersdobler A, Haese A, Huland H. Influence of nerve-sparing procedure during radical prostatectomy on margin status and biochemical failure. Eur Urol 2005; 47: 176 84.

15. Bañez LL, Sun L, Trock BJ, et al. Body mass index and prostate specific antigen as predictors of adverse pathology and biochemica recurrence after prostatectomy. J Urol 2009; 182: 491-8.

16. Preston MA, Carrière M, Raju G, et al. The prognostic significance of capsular incision into tumor during radical prostatectomy. Eur Urol 2011; 59: 613-8.

17. Freedland SJ, Aronson W, Presti JC Jr, Kane CJ, Terris MK, Elashoff D, Amling CL. Should a positive surgical margin following radical prostatectomy be pathological stage T2 or T3? Results from the SEARCH database. J Urol 2003; 169: 2142-6.

18. Alkhateeb S, Alibhai S, Fleshner N, et al. Impact of positive surgical margins after radical prostatectomy differs by disease risk group. J Urol 2010; 183: 145-50.

19. Moul JW. Post-radical prostatectomy management options for positive surgical margins: argument for observation. Urol Oncol 2009; 27: 92-6.

20. Jayachandran J, Bañez LL, Levy DE, et al. Risk stratification for biochemical recurrence in men with positive surgical margins or extracapsular disease after radical prostatectomy: results from the SEARCH database. J Urol 2008; 179: 1791-6.

21. Teh BS, Bastasch MD, Mai WY, Kattan MW, Butler EB, Kadmon D. Long-term benefits of elective radiotherapy after prostatectomy for patients with positive surgical margins. J Urol 2006; 175: 2097-102.

22. Thompson IM, Tangen CM, Paradelo J, et al. Adjuvant radiotherapy for pathological T3NOMO prostate cancer significantly reduces risk of metastases and improves survival: long-term follow up of a randomized clinical trial. J Urol 2009; 183: 956-62.

23. Van der Kwast TH, Bolla M, Van Poppel H, et al. Identification of patients with prostate cancer who benefit from immediate postoperative radiotherapy: EORTC 22911. J Clin Oncol 2007; 25: 4178-86.

24. Wright JL, Dalkin BL, True LD, Ellis WJ, Stanford JL, Lange PH, Lin DW. Positive surgical margins at radical prostatectomy predict prostate cancer specific mortality. J Urol 2010; 183: 2213-8.

25. Touijer K, Kuroiwa K, Vickers A, Reuter VE, Hricak H, Scardino PT, GuilIonneau B. Impact of a multidisciplinary continuous quality improvement program on the positive surgical margin rate after laparoscopic radical prostatectomy. Eur Urol 2006; 49: 853-8.

\section{Address for correspondence}

Marta Biedka MD, PhD

Clinic of Oncology and Brachytherapy Ludwik Rydygier Collegium Medicum Romanowskiej 2

85-796 Bydgoszcz, Poland

e-mail: martabiedka@tlen.pl

Submitted: 7.01 .2013

Accepted: $\quad 20.02 .2013$ 